\title{
FEROMÔNIOS DE AGREGAÇÃO EM CURCULIONIDAE (INSECTA: COLEOPTERA) E SUA IMPLICAÇÃO TAXONÔMICA
}

\author{
Bianca Giuliano Ambrogi, Diogo Montes Vidal e Paulo Henrique Gorgatti Zarbin* \\ Departamento de Química, Universidade Federal do Paraná, CP 19081, 81531-990 Curitiba - PR, Brasil \\ Germano Henrique Rosado-Neto \\ Departamento de Zoologia, Universidade Federal do Paraná, CP 19020, 81531-980 Curitiba - PR, Brasil
}

Recebido em 26/9/08; aceito em 24/4/09; publicado na web em 7/10/09

\begin{abstract}
AGGREGATION PHEROMONE IN CURCULIONIDAE (INSECTA: COLEOPTERA) AND THEIR TAXONOMIC IMPLICATION. The pheromones of the weevils has been the most studied and used so far for Coleoptera species. The majority of reported weevil pheromones is produced by males and usually attract both sexes. The identified pheromone compounds are classified in two categories: ten-carbon compounds with terpenoid branching and compounds of various sizes, apparently of fatty-acid origin. These pheromone structural categories are consistent within subfamilies. This review aims to give an overview of the aggregation pheromones identified for Curculionidae pests, describing the relationship of the molecules structural pattern among subfamilies, and propose an identification key based on the structure of the pheromone components.
\end{abstract}

Keywords: weevils; pheromone structure; identification key.

\section{INTRODUÇÃO}

Os curculionídeos são besouros da superfamília Curculionoidea, com cerca de 60.000 espécies descritas. ${ }^{1}$ Os adultos da família Curculionidae são facilmente reconhecidos pela presença de uma cabeça prolongada em um rostro de comprimento muito variável, assim como antenas genículo-clavadas ${ }^{2}$ (Figura 1). Os curculionídeos, em sua grande maioria, são fitófagos, tanto na fase larval como na adulta. ${ }^{3}$ Muitas espécies são importantes pragas de diversas culturas, bem como de grãos armazenados, e a necessidade constante de medidas de controle mais efetivas e seguras ao meio ambiente tem exigido da comunidade científica a busca por novos métodos de controle para minimizar o impacto desses insetos. Como em outros grupos de insetos, esta necessidade tem aumentado a investigação do uso de feromônios como mais uma ferramenta no manejo dessas pragas. Um trabalho extensivo tem sido feito na identificação de feromônios e seu uso dentro da família Curculionidae, ${ }^{4}$ exemplificado pelas décadas de pesquisa conduzidas com o bicudo do algodoeiro, Anthonomus grandis. ${ }^{5}$ Seguindo a identificação de quatro componentes do feromônio de agregação de $A$. grandis,${ }^{6}$ feromônios de várias espécies de curculionídeos têm sido identificados.

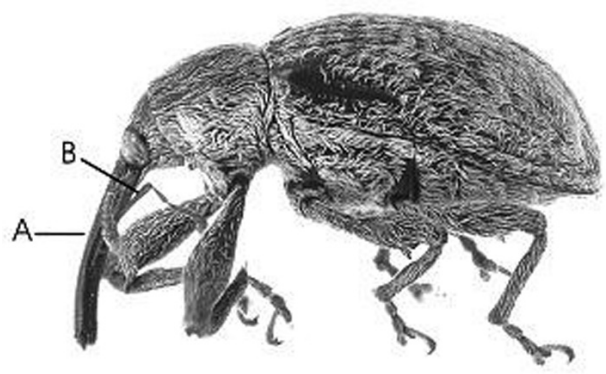

Figura 1. Vista lateral de um curculionídeo típico (Anthonomus grandis) A - Rostro, B - Antenas genículo-clavadas (Foto: Projeto Táxon Line - Entomóloga Norma Giambarresi Ganho)

*e-mail: pzarbin@quimica.ufpr.br
A maioria dos feromônios relatada em curculionídeos é produzida pelos machos e usualmente atrai ambos os sexos, por isso são conhecidos como feromônios de agregação. Entretanto, alguns feromônios também têm sido verificados em fêmeas. Estes incluem feromônios sexuais de longa-distância, que atraem principalmente machos, curta-distância ou feromônio de contato, que permitem reconhecer coespecíficos e feromônio de oviposição, que algumas fêmeas usam para marcar os hospedeiros, nos quais elas colocam seus ovos. Finalmente, existem exemplos de curculionídeos utilizando feromônios de outras espécies de besouros, como cairomônios. ${ }^{4}$

Em virtude de seus hábitos de vida, o estudo comportamental de curculionídeos é relativamente complexo. Uma alternativa que evita muitos problemas com bioensaios e acelera o processo de identificação é a cromatografia gasosa acoplada à eletroantenografia (CG-EAG). ${ }^{7}$ Com a CG-EAG tem sido possível identificar exatamente quais compostos têm atividade sensorial nos insetos. Esta informação, combinada com comparações dos cromatogramas de voláteis coletados de machos e fêmeas, permite a identificação dos compostos específicos de cada sexo. Os compostos que constituem os feromônios de curculionídeos correspondem, basicamente, a duas classes: monoterpenoides (majoritariamente cíclicos) (Figura 2) e possíveis derivados de ácidos graxos (álcoois, cetonas, e ésteres de cadeia metilramificadas e com número variável de carbono) $)^{4,8}$ (Figura 3).

O objetivo desta revisão é dar uma visão geral sobre os feromônios de agregação presentes em algumas espécies de Curculionidae de importância econômica, relacionando o aspecto estrutural dos feromônios com as diferentes subfamílias e tribos e demonstrar algumas aplicações dessa relação nos estudos taxonômicos.

\section{FEROMÔNIOS DE CURCULIONÍDEOS}

As espécies de Curculionidae, subfamílias, tribos e os compostos já identificados estão listados na Tabela 1. Estas espécies pertencem a 6 subfamílias e 10 tribos. Os nomes dos táxons e das categorias estão de acordo com as propostas de classificação mais recentes para os curculionídeos ${ }^{1,9}$. As descrições estão ordenadas alfabeticamente pela subfamília e as estruturas químicas estão apresentadas nas Figuras 2 e 3. 
Tabela 1. Espécies pragas de Curculionidae e seus feromônios de agregação. Verificar a estrutura dos compostos nas Figuras 2 e 3

\begin{tabular}{|c|c|c|c|}
\hline Espécies & Subfamília & Tribo & Compostos \\
\hline Pseudopiazurus obesus & Conoderinae & Piazurini & 1,2 \\
\hline Anthonomus eugenii & Curculioninae & Anthonomini & $3,4,5,6,7,8$ \\
\hline Anthonomus grandis & Curculioninae & Anthonomini & $2,3,5,6$ \\
\hline Anthonomus rubi & Curculioninae & Anthonomini & $2,3,5,6,9,10$ \\
\hline Curculio caryae & Curculioninae & Curculionini & $2,3,5,6$ \\
\hline Cosmopolites sordidus & Dryophthorinae & Rhynchophorini & $12,13,14,15$ \\
\hline Dynamis borassi & Dryophthorinae & Rhynchophorini & 16 \\
\hline Metamasius hemipterus & Dryophthorinae & Rhynchophorini & $16,17,18,19,20$ \\
\hline Metamasius hemipterus sericeus & Dryophthorinae & Rhynchophorini & $16,17,18,21,22,23,24,25$ \\
\hline Metamasius spinolae & Dryophthorinae & Rhynchophorini & $23,26,27$ \\
\hline Rhabdoscelus obscurus & Dryophthorinae & Rhynchophorini & $17,18,28$ \\
\hline Rhynchophorus bilineatus & Dryophthorinae & Rhynchophorini & 16 \\
\hline Rhynchophorus cruentatus & Dryophthorinae & Rhynchophorini & 29 \\
\hline Rhynchophorus ferrugineus & Dryophthorinae & Rhynchophorini & 16,22 \\
\hline Rhynchophorus palmarum & Dryophthorinae & Rhynchophorini & 28 \\
\hline Rhynchophorus phoenicis & Dryophthorinae & Rhynchophorini & 30 \\
\hline Rhynchophorus vulneratus & Dryophthorinae & Rhynchophorini & $16,22,30$ \\
\hline Scyphophorus acupunctatus & Dryophthorinae & Rhynchophorini & 17,18 \\
\hline Sitophilus oryzae & Dryophthorinae & Rhynchophorini & 31 \\
\hline Sitophilus zeamais & Dryophthorinae & Rhynchophorini & 31 \\
\hline Sitophilus granarius & Dryophthorinae & Rhynchophorini & 31,32 \\
\hline Sphenophorus levis & Dryophthorinae & Rhynchophorini & 18 \\
\hline Diaprepes abbreviatus & Entiminae & Eustylini & Não identificado \\
\hline Sitona lineatus & Entiminae & Sitonini & 33 \\
\hline Bothynoderes punctiventris & Lixinae & Cleonini & 5,6 \\
\hline Conotrachelus nenuphar & Molytinae & Conotrachelini & 11 \\
\hline Pissodes spp. & Molytinae & Pissodini & 1,2 \\
\hline Sternechus subsignatus & Molytinae & Sternechini & Não identificado \\
\hline
\end{tabular}

\section{Subfamília Conoderinae}

\section{Pseudopiazurus obesus}

Também conhecido como broca do mamoeiro é considerada uma importante praga na cultura do mamão no Nordeste do Brasil. ${ }^{10}$ Testes em laboratório utilizando olfatômetro em Y demonstraram que tanto machos como fêmeas são atraídos para uma combinação dos voláteis emitidos pelos machos da espécie e pela planta hospedeira. ${ }^{11}$ Análises comparativas dos voláteis emitidos pelos machos e fêmeas revelaram três compostos macho-específicos. O composto majoritário foi identificado como o 2-((1R, 2S)-1-metil-2-(prop-1-en-2-il)ciclobutil)acetaldeído (1c) (grandisal) e o intermediário como o 2-((1R,2S)-1-metil-2-(prop-1-en-2il)ciclobutil)etanol (2c) (grandisol) (Figura 2), enquanto que a estrutura química do componente minoritário ainda não foi determinada. ${ }^{11}$

\section{Subfamília Curculioninae}

Anthonomus eugenii

Este inseto é uma importante praga de pimenta e pimentões (Capsicum spp.) no sul dos Estados Unidos, México e na América Central. ${ }^{12}$ Trabalhos de campo forneceram a primeira evidência que machos de A. eugenii produzem um feromônio de agregação. ${ }^{13}$ A identificação deste feromônio foi realizada em 1994 por Eller e colaboradore ${ }^{14}$ como sendo uma mistura dos seguintes álcoois: (Z)-2-(3,3-dimetilcicloexilideno)etanol (3) e seu isômero (E)-2-(3,3dimetilcicloexilideno)etanol (4), os respectivos aldeídos (Z)-2-(3,3cicloexilideno)acetalaldeído (5) e (E)-2-(3,3-cicloexilideno)acetalaldeído (6), o ácido (E)-3,7-dimetilocta-2,6-dienoico (ácido gerânico) (7) e o (E)-3,7-dimetilocta-2,6-dien-1-ol (geraniol) (8) (Figura 2). 


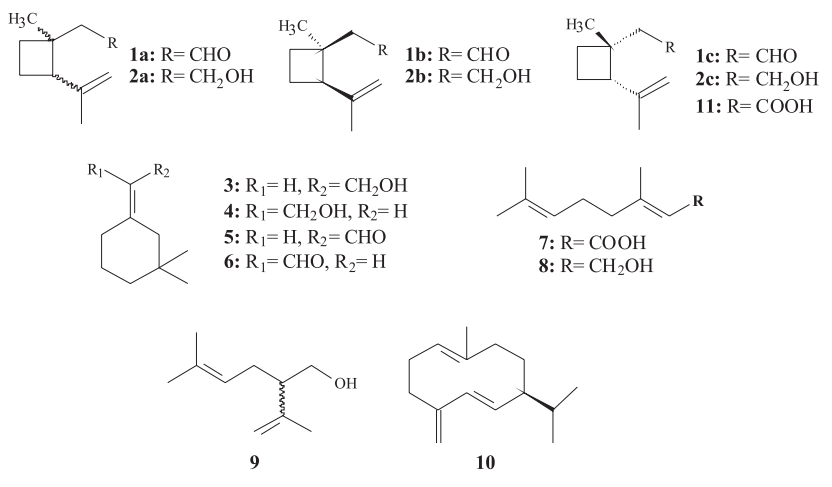

Figura 2.

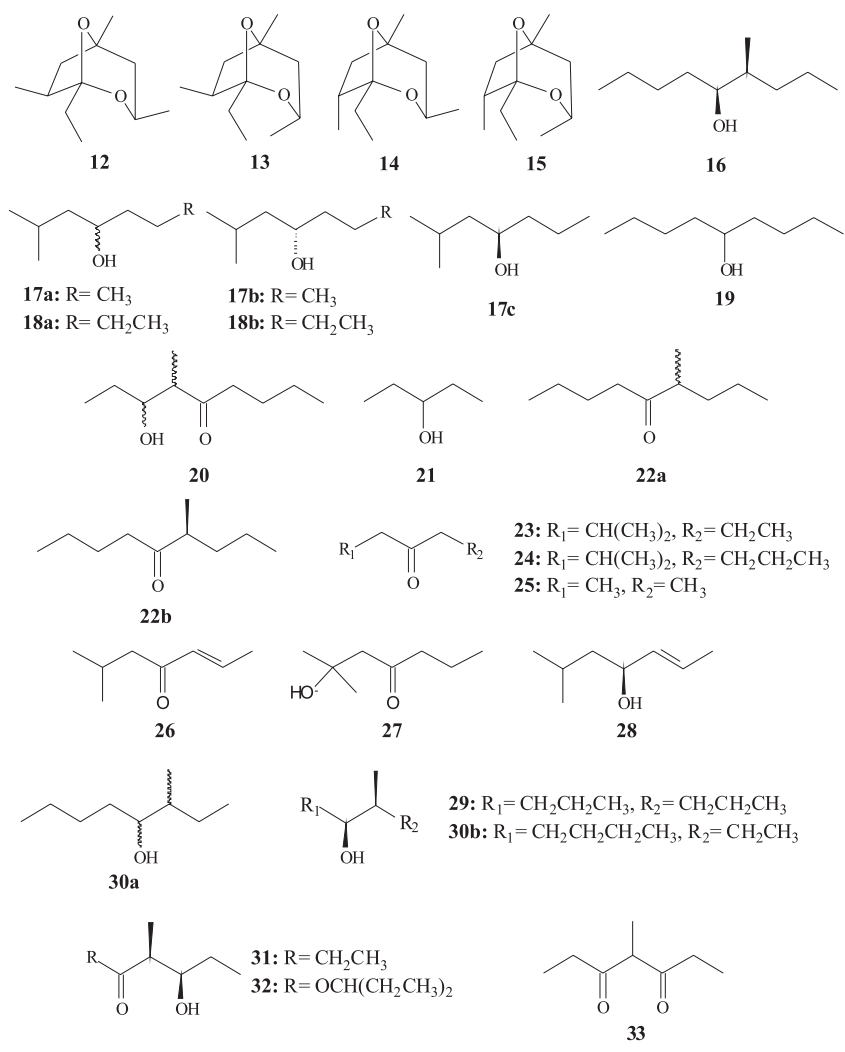

Figura 3.

\section{Anthonomus grandis}

Popularmente conhecido como bicudo-do-algodoeiro este inseto é considerada a principal praga dos algodoeiros nas Américas, pois provoca queda anormal dos botões florais e flores, impedindo a abertura normal das maçãs. ${ }^{15}$ Foi o primeiro curculionídeo a ter o feromônio estudado, o qual é liberado pelos machos e constituído de quatro componentes; os álcoois $\mathbf{2 a}$ e $\mathbf{3}$ e os aldeídos $\mathbf{5}$ e $\mathbf{6}^{6}$ (Figura 2). A mistura desses quatro componentes foi ativa em bioensaios de laboratório. ${ }^{6}$ Formulações sintéticas do feromônio atraíram tanto machos como fêmeas no campo. ${ }^{16} \mathrm{~A}$ configuração absoluta do grandisol foi estabelecida como sendo $(1 R, 2 S)(\mathbf{2 c}) \cdot{ }^{17}$ Este feromônio tem sido amplamente comercializado para o monitoramento e controle deste inseto. ${ }^{18} A$. eugenii e A. grandis apresentam três compostos feromonais em comum.

\section{Anthonomus rubi}

Considerado a principal praga de cultivos de morango na Europa. ${ }^{19}$ Como as outras espécies estudadas dentro dessa mesma subfamília, os machos de A. rubi também produzem componentes do feromônio do bicudo-do-algodoeiro, A. grandis. Os compostos feromonais encontrados nesta espécie foram o grandisol $\mathbf{2 a}$, o álcool 3, o 5-metil-2-(prop-1-en-2-il)-hex-4-en-1-ol (lavandulol) (9) e o $(S, 1 Z, 6 E)$-8-isopropil-1-metil-5-metilenociclodeca-1,6-dieno (germacreno D) (10) ${ }^{20}$ (Figura 2). Novamente para esta espécie, a estereoquímica absoluta do grandisol foi estabelecida como sendo $(1 R, 2 S)(\mathbf{2 c})$, enquanto que para o lavandulol, a configuração absoluta ainda não foi determinada. Pequenas quantidades dos aldeídos 5 e 6 também foram encontradas. ${ }^{20}$ Testes de campo mostraram que uma combinação somente dos compostos $\mathbf{2 a}$ (racêmico), $\mathbf{3}$ e $\mathbf{9}$ foram suficientes para atrair machos e fêmeas dessa espécie. A adição de pequenas quantidades dos aldeídos e dos demais compostos não aumentou as capturas. ${ }^{20}$

\section{Curculio caryae}

É uma das mais importantes pragas dos cultivos de pecã nos Estados Unidos. ${ }^{21}$ No final do verão, os adultos atacam os frutos em maturação, danificando-os por meio da alimentação ou da postura. ${ }^{22}$ Os estudos com esse inseto iniciaram em 1971, quando van Cleave e Harp $^{23}$ demonstraram que gaiolas com fêmeas dessa espécie atraíam uma maior quantidade de adultos de ambos os sexos, quando comparadas com gaiolas contendo machos. Vários estudos nesse sentido foram feitos posteriormente, mas nenhum dos testes utilizado foi suficiente para permitir avaliações estatísticas satisfatórias. O estudo do comportamento desse inseto foi dificultado por uma série de razões e, somente em 1997, Hedin e colaboradores ${ }^{24}$ identificaram o feromônio produzido pelos machos de C. caryae como sendo uma mistura de 5 componentes: isômeros cis e trans do grandisol $\mathbf{2 a}$, álcool $\mathbf{3}$ e os aldeídos 5 e 6 (Figura 2). Testes em olfatômetro demonstraram que os compostos são mais atrativos para as fêmeas que para os machos. ${ }^{24}$ Novamente, esses compostos ocorrem também em várias espécies de Curculionidae. Entretanto, em todos os casos, a liberação e a proporção de cada composto são diferentes para as diferentes espécies.

\section{Subfamília Dryophthorinae}

\section{Cosmopolites sordidus}

Cosmopolites sordidus, vulgarmente conhecido por moleque-dabananeira, é amplamente distribuído por todas as regiões do Brasil e é uma das principais pragas da bananeira. ${ }^{15}$ Bioensaios utilizando olfatômetro e CG-EAG demonstraram a existência de um feromônio de agregação produzido pelos machos da espécie. ${ }^{25}$ Subsequentemente, Beauhaire e colaboradore ${ }^{26}$ detectaram a presença de 6 compostos específicos dos machos e identificaram o majoritário desta mistura como sendo o 1-etil-3,5,7-trimetil-2,8-dioxa-biciclo[3.2.1]octano (sordidina) (12) (Figura 3). Posteriormente, Mori e colaboradores ${ }^{27}$ determinaram a estereoquímica absoluta deste composto, como o isômero $(1 S, 3 R, 5 R, 7 S)$. Ndiege e colaboradores ${ }^{28}$ e Jayaraman e colaboradores ${ }^{28}$ desenvolveram uma síntese em grande escala da mistura racêmica da sordidina, o que tornou possível a realização de testes de campo. Armadilhas contendo a mistura dos quatro diastereoisômeros (12-15) (Figura 3) foram atrativas tanto para machos como para fêmeas. ${ }^{28}$ Atualmente, este feromônio é comercializado com o nome de Cosmolure ${ }^{\circledR}$ e tem sido amplamente empregado em técnicas de monitoramento e controle dessa praga. ${ }^{29}$

\section{Dynamis borassi}

Este inseto é considerado uma importante praga de palmáceas e está amplamente distribuído na América Central e do Sul. ${ }^{30}$ GiblinDavis e colaboradores ${ }^{31}$ identificaram o feromônio de agregação produzido por machos dessa espécie como sendo o $(4 S, 5 S)$-4metilnonan-5-ol (16) (Figura 3). Análises em CG-EAG revelaram que machos e fêmeas respondem também para o feromônio de algumas 
espécies simpátricas do gênero Rhynchophorus. ${ }^{31}$ Em experimentos de campo, as fêmeas foram igualmente atraídas para armadilhas contendo cana-de-açúcar e o composto 16, ou para outros compostos comumente encontrados nesta subfamília. Entretanto, houve uma maior captura de machos em armadilhas contendo somente o composto 16 e cana-de-açúcar. ${ }^{31}$

\section{Metamasius hemipterus}

O M. hemipterus é uma importante praga nos cultivos de banana, abacaxi, cana-de-açúcar e em várias espécies de palmáceas. ${ }^{32}$ Ocorre desde os Estados Unidos até o Uruguai, norte da Argentina e na África. ${ }^{33}$ Comparações entre voláteis coletados de machos e fêmeas revelaram a presença de um feromônio produzido pelos machos, que consiste de uma mistura de 5 compostos: o álcool 16 (composto majoritário), 2-metil-heptan-4-ol (17a), 2-metiloctan-4-ol (18a), nonan-5-ol (19) e 3-hidroxi-4-metilnonan-5-ona (20) (Figura 3). ${ }^{34,35}$ Análises eletroantenográficas revelaram respostas significativas tanto de machos quanto de fêmeas para os compostos 16 a $19 .{ }^{35}$ As estruturas químicas dos componentes feromonais e a resposta comportamental causada pelos mesmos são semelhantes àquelas já observadas em outras espécies de rincoforídeos. ${ }^{35} \mathrm{~A}$ configuração absoluta do composto majoritário que ocorre naturalmente foi determinada como (4S, 5S)-16 ${ }^{36}$ (Figura 3). Entretanto, no caso do composto $\mathbf{1 7}$ constatou-se a presença de ambos os isômeros (17b e 17c). No campo, foi demonstrado que a combinação de cana-de-açúcar e o composto majoritário 16 foi atrativa para machos e fêmeas. A adição dos álcoois 17a e 18a a essa mistura aumentou o número de insetos capturados nas armadilhas. Por outro lado, a adição dos compostos 19 e 20 não aumentou a atração dos insetos. ${ }^{37}$

Perez e colaboradores ${ }^{38}$ verificaram a presença de um feromônio de agregação produzido pelos machos da subespécie $M$. hemipterus sericeus. Eles encontraram todos os compostos já descritos por Ramirez-Lucas e colaboradores, ${ }^{35}$ exceto os compostos 19 e 20. Além desses compostos, encontraram também o pentan-3-ol (21), 4-metilnonan-5-ona (22a), 2-metil-heptan-4-ona (23), 2-metiloctan4-ona (24) e pentan-3-ona (25) (Figura 3). Não se sabe ainda se essas diferenças químicas encontradas na subespécie são devidas à metodologia empregada ou se existem variações geográficas entre os curculionídeos estudados. ${ }^{39}$

\section{Metamasius spinolae}

O dano causado por este inseto é um fator limitante para a produção comercial de palma forrageira no México. As larvas vivem nas hastes das plantas, formando galerias e, frequentemente, destroem toda a planta devido à queda dos ramos. ${ }^{15,40}$ Tafoya e colaboradores, ${ }^{41}$ por meio de testes de laboratório e campo, demonstraram a existência de um feromônio de agregação produzido pelos machos, que é atrativo para ambos os sexos em M. spinolae. Análises posteriores em cromatógrafo gasoso acoplado a um espectrômetro de massas (CG-MS) do extrato natural de machos revelaram a presença de três compostos principais: a cetona $\mathbf{2 3},(E)$-6-metil-hept-2-en-4-ona (26) e 2-hidroxi-2-metil-heptan-4-ona (27) (Figura 3), em ordem crescente de abundância. ${ }^{42}$ Os compostos 26 e 27 ainda não tinham sido identificados em insetos. Testes de campo mostraram maior atratividade dos insetos para as armadilhas contendo os compostos 23 e 26 e para mistura dos três compostos $(\mathbf{2 3}, \mathbf{2 6}$ e $\mathbf{2 7})$, mais a planta hospedeira.

\section{Rhabdoscelus obscurus}

Este inseto, considerado praga de cana e palmáceas ornamentais, é nativo da área austromalásia. ${ }^{43}$ Testes em laboratório e em campo com $R$. obscurus deram a primeira evidência que machos produzem um feromônio de agregação que é atrativo principalmente para as fêmeas. ${ }^{44}$ Giblin-Davis e colaboradores ${ }^{45}$ identificaram o feromônio de populações de machos de R. obscurus do Havaí como o álcool 18a e os compostos feromonais de populações de $R$. obscurus da Austrália como uma mistura dos compostos 18a, (E)-6-metil-hept-2-en-4-ol (28a) e 17a (Figura 3). Posteriormente, testes de campo em populações da ilha de Guam (Oceania) demonstraram que $R$. obscurus foi mais atraído para armadilhas contendo o feromônio das populações da Austrália do que as que continham o feromônio das populações do Havaí. ${ }^{46}$

\section{Rhynchophorus bilineatus}

O gênero Rhynchophorus consiste de 10 espécies, dentre as quais 7 são consideradas pragas de coco. ${ }^{30}$ Oehlschlager e colaboradores ${ }^{47}$ identificaram o feromônio de agregação dessa espécie como sendo o álcool 16 (Figura 3). Este composto também faz parte do feromônio de agregação de outras duas espécies de Rhynchophorus, $R$. ferrugineus e $R$. vulneratus, além de $M$. hemipterus.

\section{Rhynchophorus cruentatus}

Esta espécie ocorre na América do Norte e é uma praga importante de palmáceas ornamentais, principalmente quando elas estão estressadas ou danificadas. Os machos são os responsáveis pela liberação de um feromônio de agregação que é altamente atrativo para ambos os sexos quando combinado com os voláteis da planta hospedeira. ${ }^{48}$ Em 1994, Weissling e colaboradores ${ }^{49}$ identificaram o único composto macho-específico presente no feromônio de agregação de $R$. cruentatus como sendo o (4S, 5S)-5-metiloctan-4-ol (29) (Figura 3).

\section{Rhynchophorus ferrugineus e Rhynchophorus vulneratus}

$R$. ferrugineus e $R$. vulneratus são duas espécies simpátricas, importantes pragas de palmáceas no sul e sudeste da Ásia. ${ }^{50}$ Hallet e colaboradores ${ }^{51}$ descobriram em ambas as espécies a presença de dois compostos produzidos especificamente pelos machos, o álcool 16 e a cetona 22a (Figura 3). Nessa mesma época, Rochat e colaboradores ${ }^{52}$ encontraram em $R$. vulneratus a presença de um composto em menor quantidade, o 3-metiloctan-4-ol (30a) (Figura 3).

A falta de diferença pronunciada na composição feromonal dessas duas espécies sugere que a quiralidade dos compostos determina a especificidade da comunicação química. ${ }^{53}$ Entretanto, análises posteriores para verificar a estereoquímica desses compostos revelaram que as duas espécies produzem o $(4 S, 5 S)-\mathbf{1 6}^{36,54}$ e o $(S)$-4-metilnonan-5-ona (22b). ${ }^{54}$ Experimentos de campo demonstraram que ambas respondem igualmente ao isômero $(4 S, 5 S)$-16 e para a mistura racêmica, mas não respondem ao isômero $(4 R, 5 R)^{54}$. A cetona $22 \mathbf{b}$ não influenciou na atividade do composto majoritário. ${ }^{54}$

\section{Rhynchophorus palmarum}

$\mathrm{O}$ R. palmarum é uma praga importante das plantações de coco na Venezuela, México, Brasil e áreas do Caribe. ${ }^{55}$ As larvas deste inseto penetram na planta, alimentando-se do pecíolo da folha e da região meristemática. Entretanto, os adultos são o maior problema, pois transportam o nematoide Bursaphelencus cocophilus, agente causador da doença conhecida por anel vermelho. ${ }^{56}$ Esta doença é letal para o coqueiro e outras palmeiras.

Trabalhos de campo e laboratório forneceram a primeira evidência de que machos de $R$. palmarum na presença da planta hospedeira produzem um feromônio de agregação que atrai tanto machos como fêmeas. ${ }^{57}$ Posteriormente Rochat e colaboradores ${ }^{58}$ identificaram a substância 28a (Figura 3) como o principal composto responsável pela atratividade de machos de R. palmarum aos coespecíficos. Este composto foi denominado rincoforol. A estereoquímica absoluta do rincoforol natural foi definida como sendo o $(S, E)$-6-metilept-2en-4-ol (28b). ${ }^{59}$ Testes de campo demonstraram que o isômero $(R)$ não possui atividade inibitória sobre o isômero ativo $(S)$, permitindo assim a utilização no campo da mistura racêmica, cuja síntese é 
mais simples. ${ }^{60}$ Testes de campo também têm demonstrado que o ( \pm )-rincoforol 28a apresenta uma maior eficiência quando associado com pedaços de cana, ${ }^{61}$ estipe do coqueiro ${ }^{62}$ ou frutos do abacaxi. ${ }^{63}$

\section{Rhynchophorus phoenicis}

Rochat e colaboradores ${ }^{34}$ e Gries e colaboradores, ${ }^{64}$ em 1993 isolaram e identificaram um composto produzido especificamente pelos machos dessa espécie, o álcool 30a (Figura 3). A configuração absoluta desse composto foi determinada como $(3 S, 4 S)(\mathbf{3 0 b}){ }^{65,66}$ Testes de campo revelaram que o isômero $(3 S, 4 S)$ foi mais ativo, mas que a presença do $(3 R, 4 R)$ não prejudicou as capturas. ${ }^{66,67}$

\section{Scyphophorus acupunctatus}

Em muitos países, esse curculionídeo é considerado a principal praga nos cultivos de agaves. Essa espécie é amplamente distribuída, sendo encontrada desde o sul dos Estados Unidos até o Brasil, além do Caribe, Havaí, Bornéu, Java e Austrália. ${ }^{68}$ Testes em laboratório e em campo demonstraram que machos dessa espécie produzem um feromônio de agregação que atrai ambos os sexos e que essa atração aumenta na presença da planta hospedeira. ${ }^{69}$ Machos e fêmeas de $S$. acupunctatus foram atraídos para os compostos 17a e 18a (Figura 3), os quais são componentes do feromônio de agregação de M. hemipterus. ${ }^{69}$ Recentemente análises em CG-EAG revelaram quatro compostos produzidos pelos machos, os álcoois 17a e 18a e as cetonas correspondentes $\mathbf{2 3}$ e $\mathbf{2 4} \cdot{ }^{70}$ Experimentos de laboratório e campo demonstraram que os compostos $\mathbf{2 3}$ e $\mathbf{2 4}$ foram tão eficientes na captura dos insetos quanto as armadilhas carregadas com a mistura completa (17a, 18a, 23 e 24$).^{70}$

\section{Sitophilus spp}

As espécies inclusas neste gênero, também chamadas de gorgulhos dos cereais, são consideradas pragas de grãos armazenados, como arroz, milho, trigo e cevada. ${ }^{15}$ Os feromônios de agregação produzidos pelos machos ocorrem em $S$. oryzae, $S$. zeamais e $S$. granarius. ${ }^{71}$ Walgenbach e colaboradores ${ }^{72}$ identificaram o feromônio de agregação de $S$. oryzae e $S$. zeamais como o $(4 S, 5 R)$-5-hidroxi4-metil-heptan-3-ona (31) (Figura 3), comumente conhecido como sitophinona. ${ }^{73} \mathrm{O}$ feromônio de $S$. granarius foi identificado como ( $2 S$, $3 R$ )-2-metil-3-hidroxipentanoato de 1-etilpropila (32) ${ }^{74}$ (Figura 3), comumente conhecido como sitophilato. Análises eletroantenográficas detectaram respostas para mais um componente em $S$. granarius, mas que ainda não foi identificado. ${ }^{75}$ Vários autores têm demonstrado que $S$. granarius é atraído também para a sitophinona 31, mas que $S$. oryzae e $S$. zeamais não são atraídos para o sitophilato $32 .{ }^{76}$ Wakefielf e colaboradores ${ }^{77}$ sugeriram uma isca contendo somente o composto 31 para monitoramento das 3 espécies de Sitophilus.

\section{Sphenophorus levis}

É uma importante praga da cana-de-açúcar e tem sido encontrado principalmente no norte da Argentina, Paraguai e no Brasil. ${ }^{78}$ Zarbin e colaboradores ${ }^{79}$ identificaram o álcool $(S)$-2-metiloctan-4-ol (18b) (Figura 3), o qual é produzido especificamente pelos machos dessa espécie. Testes preliminares em laboratório utilizando a mistura racêmica sugeriram que este composto provoca um comportamento de agregação entre machos e fêmeas da espécie. ${ }^{79} \mathrm{O}$ composto 18a já foi previamente identificado como componente do feromônio de agregação de várias espécies da subfamília Dryophthorinae.

\section{Subfamília Entiminae}

\section{Diaprepes abbreviatus}

É a principal praga de cana nas ilhas caribenhas e uma importante praga de citros na Flórida. ${ }^{80}$ Schroeder ${ }^{81}$ evidenciou um feromônio produzido pelos machos, que atrai as fêmeas, e um feromônio produzido pelas fêmeas, que atrai os machos. Posteriormente, Beavers e colaboradores $^{82}$ sugeriram que o odor de plantas jovens de citros é o principal responsável pela agregação desses insetos e que as fezes no local de agregação podem servir como um atraente secundário para o sexo oposto. Jones e Schroeder ${ }^{83}$ demonstraram que um feromônio produzido pelo macho, liberado nas fezes, atrai ambos os sexos de D. abbreviatus. Testes em laboratório utilizando olfatômetro em Y demonstraram que machos e fêmeas foram atraídos para a planta hospedeira, para machos ou fêmeas isoladamente e para as fezes de machos e fêmeas. ${ }^{84} \mathrm{~A}$ evidência de comunicação química nessa espécie é contraditória e a identificação dos compostos responsáveis por essa comunicação ainda não existe.

\section{Sitona lineatus}

Estes insetos são importantes pragas de leguminosas e estão distribuídos na Europa e América do Norte. Os danos mais sérios são causados pelas larvas, que destroem os nódulos radiculares, diminuindo, dessa maneira, a capacidade fixadora de nitrogênio das plantas. Blight e colaboradores ${ }^{85}$ e Blight e Wadhams ${ }^{86}$ identificaram o feromônio de agregação presente nos machos dessa espécie, como sendo o 4-metilheptano-3,5-diona (33) (Figura 3). Posteriormente, esse composto foi sintetizado e mostrou atrair ambos os sexos no campo. ${ }^{85}$

\section{Subfamília Lixinae}

\section{Bothynoderes punctiventris}

O B. punctiventris é uma importante praga de beterraba em várias regiões da Europa. ${ }^{87}$ Tóth e colaboradores ${ }^{88}$ verificaram uma atratividade de ambos os sexos de B. punctiventris em testes de campo utilizando armadilhas contendo a mistura feromonal do bicudo do algodoeiro, A. grandis, compostos 2a, 3, 5 e 6 (Figura 2). Testes posteriores revelaram que somente os aldeídos 5 e $\mathbf{6}$ são os responsáveis pela atração. ${ }^{88}$ Estudos utilizando CG-EAG mostraram que machos e fêmeas apresentaram uma maior resposta para o composto $\mathbf{5}$, sugerindo uma maior importância deste isômero na atratividade. ${ }^{88}$

\section{Subfamília Molytinae}

\section{Conotrachelus nenuphar}

Este inseto é uma praga chave nos pomares de maçã na América do Norte.$^{89}$ Eller e Bartelt ${ }^{90}$ isolaram e, subsequentemente, sintetizaram o feromônio de agregação dessa espécie, identificando-o como sendo o ácido 2-((1R, 2S)-1-metil-2-(prop-1-en-2-il)ciclobutil)acético (11) (Figura 2), comumente chamado de ácido grandisoico, por ser análogo ao grandisol 2a. Testes de campo revelaram que tanto a mistura racêmica do ácido grandisoico como os enantiômeros puros atraem machos e fêmeas da espécie..$^{90}$

\section{Pissodes spp}

Os curculionídeos do gênero Pissodes compreendem um importante grupo de pragas de coníferas na América do Norte. A primeira evidência de um feromônio de agregação em Pissodes foi obtida por Booth e Lanier, em 1974, ${ }^{91}$ quando demonstraram que machos de Pissodes approximatus juntamente com a planta hospedeira atraíram machos e fêmeas coespecíficos em testes de campo. Grandisol, 2a e seu aldeído correspondente grandisal, 1a (Figura 2), foram isolados de machos de $P$. aproximatus e P. strobi $^{92} \mathrm{e}$ mostraram ter atratividade feromonal quando foram colocados junto com a planta hospedeira. Philips e colaboradores ${ }^{93}$ observaram que machos de $P$. nemorensis também produzem os mesmos compostos feromonais 2a e 1a presentes em $P$. aproximatus e P. strobi, e que a combinação de grandisol e grandisal, juntamente com a planta hospedeira, foi a 
mais atrativa em testes de campo. Hibbard e Webster ${ }^{94}$ determinaram a quiralidade desses compostos para $P$. nemorensis e P. strobi. Ambas as espécies produzem quase que $100 \%$ do isômero $(1 R, 2 S)(\mathbf{2 c}), P$. nemorensis emite quase que $100 \%$ do enantiômero $(1 S, 2 R)(\mathbf{1 b})$, mas $P$. strobi emite aproximadamente $60 \%$ do isômero $(1 S, 2 R)(\mathbf{1 b})$ e $40 \%$ do $(1 R, 2 S)$ (1c) (Figura 2). Também foi verificada a presença de um feromônio produzido por machos em uma quarta espécie de Pissodes, $P$. schwarzi, ${ }^{95}$ mas a estrutura química correspondente ainda não foi determinada.

\section{Sternechus subsignatus}

Sternechus subsignatus, popularmente conhecido como tamanduá-da-soja, apresenta um grande potencial de dano, pois tanto as larvas quanto os adultos se alimentam da soja. ${ }^{96}$ Este inseto apresenta uma ampla distribuição, ocorrendo principalmente na região sul do Brasil. ${ }^{97}$ Recentemente Ambrogi e Zarbin ${ }^{98}$ verificaram a presença de um feromônio de agregação produzido pelos machos que atraem ambos os sexos. Análises em CG demonstraram a existência de cinco compostos específicos dos machos, mas a identificação química desses compostos ainda não foi concluída.

\section{APLICAÇÃO DOS FEROMÔNIOS DE AGREGAÇÃO NO MANEJO INTEGRADO DE PRAGAS (MIP)}

A utilização de inseticidas para o controle de curculionídeospraga não tem sido muito eficiente, uma vez que esses insetos geralmente são brocas, alimentam-se dentro de frutos, caules e sementes. Além disso, existe a necessidade de novos métodos de controle que agridam menos o meio ambiente. Devido a esses fatores, os feromônios tornam-se uma técnica bastante promissora para utilização no MIP.

Alguns dos feromônios citados anteriormente já são produzidos comercialmente e têm sido utilizados com sucesso no monitoramento e controle de pragas. A utilização de feromônios para monitoramento desses insetos facilita a determinação da dinâmica populacional, auxiliando a tomada de decisão do agricultor no manejo dos insetos. O controle de curculionídeos utilizando feromônios tem sido feito principalmente por meio de coleta massal e com grande sucesso, uma vez que os feromônios atraem ambos os sexos. Os estudos de feromônios nesta importante família são bons exemplos para direcionar futuras pesquisas na área, objetivando principalmente o desenvolvimento de novas técnicas de controle.

\section{IMPLICAÇÃO TAXONÔMICA}

Estudos relacionados à identificação de feromônios em insetos podem ser explorados não somente na síntese dos compostos para utilização no MIP, mas também podem contribuir para um melhor esclarecimento dos grupos taxonômicos, em especial na família Curculionidae, uma vez que existem muitas espécies com o feromônio já identificado.

Os curculionídeos das subfamílias Conoderinae, Curculioninae, Lixinae e Molytinae tendem a produzir monoterpenoides cíclicos como componentes feromonais (ex: grandisol 2a, Z- e $E$-octodenol 3 e 4 e os aldeídos correspondentes 5 e 6). Dentro da família Curculionidae, derivados de cicloexalidenos foram primeiramente verificados como compostos do feromônio de agregação do bicudo do algodoeiro A. grandis. Os compostos 5 e 6, presentes no feromônio de $A$. grandis também estão presentes na mistura feromonal de $A$. eugenii, B. punctiventris e C. caryae. A presença das substâncias 5 e 6 também foi demonstrada em voláteis produzidos por machos de $A$. rubi, entretanto, nessa espécie não foi atribuída atividade biológica para esses compostos. A atratividade dos compostos $\mathbf{5}$ e $\mathbf{6}$ sugere a importância das estruturas cicloexalideno na comunicação química entre as espécies destas subfamílias.

Já os insetos da subfamília Dryophthorinae e Entiminae, incluindo $D$. borassi, M. hemipterus, $R$. obscurus, seis espécies de Rhynchoporus, S. acupunctatus, três espécies de Sitophilus, S. levis e S. lineatus produzem álcoois secundários contendo de 5 a 10 carbonos com ramificações metílicas, provavelmente derivados de ácidos graxos, exceto para C. sordidus, onde 6 diastereoisômeros de um novo cetal bicíclico (sordidina) foram identificados como componentes feromonais. Além dos álcoois, algumas cetonas também são encontradas nos feromônios de algumas espécies dentro dessas subfamílias.

Assim, os compostos feromonais identificados para estas espécies mostram uma relação estrutural em concordância com a taxonomia da subfamília correspondente. Nesse contexto, foi proposta uma chave de identificação apresentada a seguir, tentando estabelecer agrupamentos com base nos feromônios de agregação já conhecidos. A chave foi elaborada por Rosado-Neto, especialista em taxonomia de Curculionidae e um dos autores deste trabalho, e inclui apenas as subfamílias cujos feromônios são conhecidos e estudados. Os caracteres morfológicos são os tradicionalmente conhecidos através da literatura taxonômica pertinente à família como, por exemplo, o trabalho mais recente sobre os Curculionoidea sul-americanos, de Marvaldi e Lanteri. ${ }^{1}$ Trata-se de uma proposta que certamente será ampliada no futuro por meio da identificação de novos compostos que permitirão um refinamento mais adequado na elaboração da chave de identificação.

1 - Escrobo curto; escapo antenal em repouso não se alojando dentro do escrobo; funículo antenal com 6 ou menos artículos; clava cônico-truncada, com suturas não visíveis e porção basal glabra e brilhante. Pigídio exposto. Componentes feromonais formados principalmente por álcoois secundários e cetonas contendo 5 a 10 átomos de carbonos com ramificações metílicas (exceto para Cosmopolites sordidus). Dryophthorinae 1'- Escrobo alongado, dirigido mais ou menos em direção ao olho ou curvado para a face ventral do rostro; escapo antenal em repouso alojando-se dentro do escrobo; clava antenal compacta, oblongo-oval ou subcilíndrica, com 3-4 artículos pubescentes e suturas distintas. Pigídio exposto ou não. Componentes feromonais formados por monoterpenoides cíclicos (exceto para Entiminae). $\quad 2$ 2 - Rostro robusto, curto e grosso; escapo antenal normalmente ultrapassando a margem anterior do olho; largura da fronte maior que a largura do olho. Premento cobrindo as maxilas ao menos parcialmente; mandíbulas largas e com cicatriz deixada pelo processo decidual, com superfície dorsal revestida por cerdas elou escamas. Entiminae 2'- Rostro alongado, subcilíndrico, quase sempre curvo; escapo antenal dirigido em direção ao olho ou para baixo do olho, mas não ultrapassando a orbital anterior; largura da fronte menor que a largura do olho. Premento não cobrindo as maxilas; mandíbulas menos largas, sem processo decidual, com superfície dorsal glabra, sem escamas e no máximo com 1 ou 2 cerdas.

3 - Palpos labiais curtos e telescopados, aparentemente com 1 segmento; metepimero visivel; garras tarsais conatas na base. Corpo cilindróide e mais ou menos alongado. Lixinae 3'- Palpos labiais alongados, não telescopados, com 3 segmentos; metepimero não visível, oculto sob as margens dos élitros; garras tarsais simples, livres ou apendiculadas, raramente conatas.

4 - Canal prosternal presente, ao menos até a margem posterior do prosterno. Olhos grandes, ocupando a maior parte da cabeça, quase sempre contíguos na fronte e inteiramente ex- 
postos quando o rostro está em repouso no canal prosternal. Mesepimero ascendente. Abdome (visto de perfil) ascendente da base até o ápice dos élitros. Tíbias posteriores com unco bem desenvolvido.

Conoderinae 4'- Canal prosternal ausente; se presente, raso e restrito à porção anterior do prosterno; neste caso os olhos são total ou parcialmente cobertos pelos lobos pós-oculares. Olhos menores, arredondados ou longitudinalmente ovais, subcontíguos. Mesepimero não ascendente. Abdome (visto de perfil) não ascendente da base ao ápice dos élitros. Tíbias posteriores com mucro normal, premucro quase sempre presente, raramente com unco.

5 - Canal prosternal ausente; neste caso, garras tarsais simples, livre (Pissodini) ou conatas (Sternechini) ou canal prosternal raso e presente apenas na porção anterior do prosterno; neste caso garras tarsais apendiculadas (Conotrachelini).

Molytinae

5'- Canal prosternal sempre ausente; neste caso as garras tarsais apendiculadas, e mandíbulas com movimento horizontal (Anthonomini) ou com movimento vertical (Curculionini).

Curculioninae

\section{AGRADECIMENTOS}

Ao CNPq, à CAPES e Fundação Araucária pelo apoio financeiro.

\section{REFERÊNCIAS}

1. Marvaldi, A. E.; Lanteri, A. A.; Rev. Chil. Hist. Nat. 2005, 78, 65.

2. Kuschel, G.; Mem. Entomol. Soc. Wash. 1995, 14, 5.

3. Vanin, S. A. Em Invertebrados Terrestres. Biodiversidade no Estado de São Paulo: Síntese do Conhecimento ao Final do Século XX; Joly, C. A.; Bicudo, C. E. M., eds.; Fundação de Amparo à Pesquisa do Estado de São Paulo: São Paulo, 1999, p. 135-140.

4. Bartelt, R. J. Em Pheromones of Non-Lepidopteran Insects Associated with Agricultural Plants; Hardie, J.; Minks, A. K., eds.; CABI Publishing: Wallingford, 1999, cap. 5.

5. Hardee, D. D.; Mitchell, E. B.; Southwest. Entomol. 1997, 22, 466.

6. Tumlinson, J. H.; Hardee, D. D.; Gueldner, R. C.; Thompson, A. C.; Hedin, P. A.; Minyard, J. P.; Science 1969, 166, 1010.

7. Perez, A. L.; Campos, Y.; Chinchilla, C. M.; Oehlschlager, A. C.; Gries, G.; Gries, R.; Giblin-Davis, R. M.; Castrillo, G.; Peña, J. E.; Duncan, R. E.; Gonzales, L. M.; Pierce, H. D. Jr; McDonald, R.; Andrade, R.; J. Chem. Ecol. 1997, 23, 869.

8. Seybold, S. J.; Vanderwel, D. Em Biosyntesis and endocrine regulation of pheromone production in the Coleoptera; Blomquist, G. J.; Vogt, R. G., eds.; Elsevier Academic Press, 2003, p. 137-200; Giblin-Davis, R. M.; Oehlschlager, A. C.; Perez, A. L.; Gries, G.; Gries, R.; Weissling, T. J.; Chinchilla, C. M.; Pena, J. E.; Hallet, R. H.; Pierce Jr, H. D.; Gonzales, L. M.; Fla. Entomol. 1996, 79, 153.

9. Wibmer, G. J.; O' Brien, C. W.; Mem. Am. Entomol. Inst. 1986, 39, 1; Alonso-Zarazaga, M. A.; Lyal, C. H. C.; A world catalogue of families and genera of Curculionoidea (Insecta: Coleoptera) (Excepting Scolytidae and Platypodidae), Entomopraxis: Barcelona, 1999.

10. Moreira, M. A. B.; Zarbin, P. H. G.; Rosado-Neto, G. H.; Barreto, M F. P.; Sobrinho, J. F.; Borges, M.; Circular Técnica, Embrapa Tabuleiros Costeiros 2003, 35.

11. Zarbin, P. H. G.; Moreira, M. A. B.; Haftmann, J.; Francke, W.; Oliveira, A. R. M.; J. Braz. Chem. Soc. 2007, 8, 1048.

12. Elmore, J. C.; Davis, A. C.; Campbell, R. E.; USDA Tech. Bull. 1934, 447.

13. Patrock, R. J.; Schuster, D. J.; Mitchell, E. R.; Fla. Entomol. 1992, 75, 138.
14. Eller, F. J.; Bartelt, R. J.; Shasha, B. S.; Schuster, D. J.; Riley, D. G.; Stansly, P. A.; Mueller, T. F.; Shuller, K. D.; Johnson, B.; Davis, J. H.; Sutherland, C. A.; J. Chem. Ecol. 1994, 20, 1537.

15. Gallo, D.; Nakano, O.; Silveira Neto, S.; Carvalho, R. P. L.; Batista, G. C.; Berti Filho, E.; Parra, J. R. P.; Zucchi, R. A.; Alves, S. B.; Vendramim, J. D.; Manual de Entomologia Agrícola, 2a ed., Ed. Agronômica "Ceres" Ltda.: São Paulo, 1988.

16. Hardee, D. D.; McKibben, G. H.; Gueldner, R. C.; Mitchell, E. B.; Tumlinson, J. H.; Cross, W. H.; J. Econ. Entomol. 1972, 65, 97.

17. Hobbs, P. D.; Magnus, P. D.; J. Am. Chem. Soc. 1976, 98, 4594; Mori, K; Tetrahedron 1978, 34, 915.

18. Hardee, D. D.; Mitchell, E. B.; Southwest. Entomol. 1997, 22, 465.

19. Cross, J. V.; Easterbrook, M. A.; IOBC/WPRS Bull. 1998, $21,81$.

20. Innocenzi, P. J.; Hall, D. R.; Cross, J. V.; J. Chem. Ecol. 2001, 27, 1203.

21. Barry, R. M.; Pecan South. 1947, 1, 33.

22. Raney, H. G.; Eikenbary, R. D.; Can. Entomol. 1968, 100, 1091.

23. Van Cleave, H. W.; Harp, J. J.; Proceedings, 64th Annual Convention of the. Southeastern Pecan Growers Association Mobile, AL., 1971.

24. Hedin, P. A.; Dollar, D. A.; Collins, J. K.; DuBois, J. G.; Mulder, P. G.; Hedger, G. H.; Smith, M. W.; Eikenbary, R. D.; J. Chem. Ecol. 1997, 23, 965 .

25. Budenberg, W. J.; Ndiege, I. O.; Karago, F. W.; J. Chem. Ecol. 1993, 19, 1905.

26. Beauhaire, J.; Ducrot, P-H.; Malosse, C.; Rochat, D.; Tetrahedron Lett. 1995, 36, 1043.

27. Mori, K.; Nakayama, T.; Takikawa, H.; Tetrahedron Lett. 1996, 21, 3741.

28. Ndiege, I. O.; Jayaraman, S.; Oehschlager, A. C.; Gonzales, L.; Alpizar, D.; Fallas, M.; Naturwissenschaften 1996, 83, 280; Jayaraman, S.; Ndiege, I. O.; Oehschlager, A. C.; Gonzales, L.; Alpizar, D.; Fallas, M.; Budenberg, W. I.; Ahuya, P.; J. Chem. Ecol. 1997, 23, 1145; Fletcher, M. T.; Moore, C. J.; Kitching, W.; Tetrahedron Lett. 1997, 38, 3475.

29. Tinzaara, W.; Dicke, M.; Van Huis, A.; Joop, J. J. A.; Gold, C. S.; Entomol. Exp. Appl. 2003, 106, 169.

30. Wattanapongsiri, A.; A revision of the genera Rhynchophorus and Dynamis (Coleoptera: Curculionidae), Department of Agricultural Science Bulletin: Bangkok, 1966, vol. 1.

31. Giblin-Davis, R. M.; Gries, R.; Gries, G.; Peña-Rojas, E.; Pinzón, I.; Peña, J. E.; Perez, A. L.; Pierce Jr., H. D.; Oehlschlager, A. C.; J. Chem. Ecol. 1997, 23, 2287.

32. Vaurie, P.; Bull. Am. Mus. Nat. Hist. 1966, 131, 211.

33. Lepesme, P.; Paulian, R.; Bull. Soc. Entomol. Fr. 1941, 46, 31.

34. Rochat, D.; Malosse, C.; Lettere, M.; Ramirez-Lucas, P.; Einhorn, J.; Zagatti, P.; Acad. Sci. Paris II 1993, 316, 1737.

35. Ramirez-Lucas, P.; Malosse, C.; Ducrot, P-H.; Lettere, M.; Zagatti, P.; Bioorg. Med. Chem. 1996, 4, 323.

36. Mori, K.; Hiromasa, K.; Malossse, C.; Rochat, D.; Liebigs Ann. Chem. 1993, 1993, 1201.

37. Ramirez-Lucas, P.; Rochat, D.; Zagatti, P.; Entomol. Exp. Appl. 1996, 80, 453.

38. Perez, A. L.; Campos, Y.; Chinchilla, C. M.; Oehlschlager, A. C.; Gries, G.; Gries, R.; Giblin-Davis, R. M.; Castrillo, G.; Peña, J. E.; Duncan, R. E.; Gonzales, L. M.; Pierce Jr., H. D.; McDonald, R.; Andrade, R.; J. Chem. Ecol. 1997, 23, 869.

39. Giblin-Davis, R. M.; Oehlschlager, A. C.; Perez, A. L.; Gries, G.; Gries, R.; Weissling, T. J.; Chinchilla, C. M.; Peña, J. E.; Hallet, R. H.; Pierce, H. D. Jr.; Gonzales, L. M.; Fla. Entomol. 1996, 79, 153.

40. Granados, D.; Castaneda, A. D.; El nopal: historia, fisiologia, genética, e importancia frutícula, Trilhas, México, 1991.

41. Tafoya, F.; Lopez-Collado, J.; Stanley, D.; Rojas, J. C.; Cibrian-Tovar, J.; Environ. Entomol. 2003, 32, 484.

42. Tafoya, F.; Whalon, M. E.; Vanderwoot, C.; Coombs, A. B.; CibrianTovar, J.; Environ. Entomol. 2007, 36, 53. 
43. Chang, V. C. S..; Tanimoto, V.; Ota, A. K.; Hawaiian Plant. Rec. 1970, 58 , 103; Halfpapp, K. H.; Storey, R.I.; Principes 1991, 35, 199; Zimmerman, E. C.; Australian weevils (Coleoptera: Curculionidae), CSIRO Australia and Entomological Society of America Publishing, 1993, vol. III.

44. Chang, V. C. S.; Curtis, G. A.; Environ. Entomol. 1972, 1, 476.

45. Giblin-Davis, R. M.; Gries, R.; Crespi, B.; Robertson, L. N.; Hara, A.H.; Gries, G.; O`Brien, C. W.; Pierce Jr., H. D.; J. Chem. Ecol. 2000, 26, 2763.

46. Muniappan, R.; Bamba, J.; Cruz, J.; Reddy, G. V. P.; Micronesica 2004, $37,57$.

47. Oehlschlager, A. C.; Prior, R. N. B.; Perez, A. L.; Gries, R.; Gries, G.; Pierce Jr., H. D.; Laup, S.; J. Chem. Ecol. 1995, 21, 1619.

48. Weissling, T. J.; Giblin-Davis, R. M.; Scheffrahn, R. N.; J. Chem. Ecol. 1993, 19, 1195

49. Weissling, T. J.; Giblin-Davis, R. M.; Gries, G.; Gries, R.; Perez, A. L.; Pierce Jr., H. D.; Oehlschlager, A. C.; J. Chem. Ecol. 1994, 20, 505

50. Sadakathulla, S.; ibid 1991, 67, 415.

51. Hallet, R. H.; Gries, G.; Gries, R.; Borden, J. H.; Czyzewska, E.; Oehlschlager, A. C.; Pierce Jr, H. D.; Angerilli, N. P. D.; Raulf, A.; Naturwissenschaften 1993, 80, 328.

52. Rochat, D. C.; Malosse, C.; Lettere, M.; Ramirez-Lucas, P.; Einhorn, J.; Zagatti, P.; C. R. de Acad. Sci. Paris. Ser. II, 1993, 316, 1737.

53. Corrêa, A. G. Em Produtos Naturais no controle de insetos, $2^{\mathrm{a}}$ ed.; Corrêa, A. G.; Vieira, P. C., orgs.; Séria de Textos da Escola de Verão em Química, Vol. III, EdUFSCar, São Carlos, 2007, p. 19.

54. Perez, A. L.; Hallet, R. H.; Gries, R.; Gries, G.; Oehlschlager, C. A.; Borden, J. H.; J. Chem. Ecol. 1996, 22, 357.

55. Hernándes, J. V.; Cerda, H.; Jaffé, K.; Sanchez, P.; Agron. Trop. 1992, $42,211$.

56. Griffith, R.; J. Agric. Soc. Trin. Tob. 1968, 68, 342.

57. Rochat, D.; González, V. A.; Mariau, D.; Villanueva, G.; Zagatti, P.; J. Chem. Ecol. 1991, 17, 1221.

58. Rochat, D.; Malosse, C.; Lettere, M.; Ducrot, P. H.; Zagatti, P.; Renou, M.; Descoins, C.; J. Chem. Ecol. 1991, 17, 2127.

59. Oehlschlager, A. C.; Pierce, H. D. Jr.; Morgan, B.; Wimalaratne, P. D. C.; Slessor, K. N.; King, G. G. S.; Gries, G.; Gries, R.; Borden, J. H.; Jiron, L. F.; Chinchilla, C. M.; Mexzan, R. G.; Naturwissenschaften 1992, 79, 134

60. Mori, K.; Ishigami, K.; Liebigs Ann. Chem. 1992, 11, 1195.

61. Rocha, D.; Descoins, C.; Malosse, C.; Nagana, P.; Zagatti, P.; Akamou, F.; Mariau, D.; Oléagineaux 1993, 48, 225.

62. Weissling, T. J.; Giblin-Davis, R. M.; Scheffrahn, R. N.; Mendonza, N. M.; Fla. Entomol. 1992, 75, 212.

63. Duarte, A. G.; Lima, I. S.; Neotropical Entomol. 2001, 30, 217.

64. Gries, G.; Gries, R.; Perez, A. L.; Oehlschlager, A. C.; Gonzales, L.M.; Pierce Jr., H. D.; Kouda-Bonafos, M.; Zebeyou, M.; Nanou, N.; Naturwissenschaften 1993, 80, 90.

65. Mori, K.; Nakayama, T.; Takikawa, H.; Liebigs Ann. Chem. 1993, 1993 , 865.

66. Perez, A. L.; Gries, G.; Gries, R.; Giblin-Davis, R. M.; Oehlschlager, A. C.; J. Chem Ecol. 1994, 20, 2653

67. Rochat, D.; Akamou, F.; Sangare, Al.; Mariau, D.; Mori, K.; C. R. Acad. Sci. Paris 1995, 318, 183

68. Vaurie, P.; Colleopt. Bull. 1971, $25,1$.

69. Ruiz-Montiel, C.; González-Hernandes, H.; Leyva, J.; Llanderal-Cazares, C.; Cruz-López, L.; Rojas, J. C.; J. Econ. Entomol. 2003, 96, 1126.
70. Ruiz-Montiel, C.; García-Coapio, G.; Rojas, J. C.; Malo, E. A.; CruzLópez, L.; Real, I.; González-Hernandes, H.; Entomol. Exp. Appl. 2008, 127, 207.

71. Moreira, M. A. B.; Zarbin, P. H. G.; Coracini, M. D. A.; Quim. Nova 2005, 28, 477.

72. Walgenbach, C. A.; Plilips, J. K.; Burkholder, W. E.; King, G. G. S.; Slessor, K, N.; Mori, K.; J. Chem. Ecol. 1987, 13, 2159.

73. Walgenbach, C. A.; Burkholder, W. E.; Environ. Entomol. 1986, 15, 733.

74. Philips, J. K.; Miller, S. P. F.; Andersen, J. F.; Fales, H. M.; Burkholder, W. E.; Tetrahedron Lett. 1987, 28, 6154; Philips, J. K.; Chong, J. M.; Andersen, J. F.; Burkholder, W. E.; Entomol. Exp. Appl. 1989, 51, 149.

75. Chambers, J.; Van Wyk, C. B.; White, P. R.; Gerrard, C.M.; Mori, K.; J. Chem. Ecol. 1996, 22, 1639.

76. Walgenbach, C. A.; Philips, J. K.; Faustini, D. L.; Burkholder, W. E.; J Chem. Ecol. 1983, 9, 831; Philips, J. K.; Walgenbach, C. A.; Klein, J. A.; Burkholder, W. E.; Schmuff, N. R.; Fales, H. M.; J. Chem. Ecol. 1985, $11,1263$.

77. Wakefield, M. E.; Bryning, G. P.; Chambers, J.; J. Stor. Prod. Research 2005, 41, 145.

78. Vanin, S. A.; A New Species of Sphenophorus Schoenher from Brazil (Coleoptera:Curculionidae, Rhynchophorinae), Ed. USP: São Paulo, 1988.

79. Zarbin, P. H. G.; Arrigoni, E. D. B.; Reckziegel, A.; Moreira, J. A.; Baraldi, P. T.; Vieira, P. C.; J. Chem. Ecol. 2003, 29, 377.

80. Cruz, C.; Segrarra, A.; Fla. Entomol. 1992, 75, 400.

81. Schroeder, W. J.; Environ. Entomol. 1981, 10, 898

82. Beavers, J. B.; McGovern, T. P.; Adler, V. E.; Environ. Entomol. 1982, $11,436$.

83. Jones, I. F.; Schroeder, W. J.; J. Econ. Entomol. 1984, 77, 334.

84. Harari, A. R.; Landolt, P. J.; J. Chem. Ecol. 1997, 23, 857.

85. Blight, M. M.; Pickett, J. A.; Smith, M. C.; Wadhams, L. J.; Naturwissenschaften 1984, 71, 480.

86. Blight, M. M.; Wadhams, L. J.; J. Chem. Ecol. 1987, 13, 733.

87. Manninger, G. A. Em Sugar-beet weevil (Cleonus Punctiventris German); Jerry, T.; Balázs, K., eds.; Akadémiai Kiadó: Budapest, 1990, p. $471-476$

88. Toth, M.; Ujváry, I.; Sivcev, I.; Imrei, Z.; Szarukán, I.; Farkas, O.; Gömöry, A.; Gács-Baitz, E.; Francke, W.; Entomol. Exp. Appl. 2007, $122,125$.

89. LeBlanc, J. P. R.; Hill, S. B.; Paradis, R. O.; Environ. Entomol. 1984, 13 , 286.

90. Eller, F .J.; Bartelt, R. J.; J. Nat. Prod. 1996, 59, 451.

91. Booth, D. C.; Lanier, G. N.; Ann. Entomol. Soc. Am. 1974, 67, 992.

92. Booth, D. C.; Phillips, T. W.; Claesson, A.; Silverstein, R. M.; Lanier, G. N.; West, J. R.; J. Chem. Ecol. 1983, 9, 1

93. Philips, T. W.; West, J. R.; Foltz, J. L.; Silverstein, R. M.; Lanier, G. N.; J. Chem. Ecol. 1984, 10, 1417.

94. Hibbard, B. E.; Webster, F. X.; J. Chem. Ecol. 1993, 19, 2129.

95. Maclaughlan, L. E.; Borden, J. H.; Price, I.; J. Entomol. Soc. British Columbia 1993, 90, 30.

96. Hoffmann-Campo, C. B.; Parra, J. R. P.; Mazzarin, R. M.; Rev. Bras. Biol. 1991, 51, 615.

97. Rosado-Neto, G. H.; An. Soc. Entomol. Brasil 1987, 16, 199.

98. Ambrogi, B. G.; Zarbin, P. H. G.; J. App. Entomol. 2008, 132, 54. 